\title{
SONOGRAPHIC DETECTION OF INTUSSCEPTION WITH MALROTATION: WAUGHS SYNDROME
}

Praveen Kumar Natesan¹, Einstein Arulraj², Moorthy Gurunathan ${ }^{3}$

\section{HOW TO CITE THIS ARTICLE:}

Praveen Kumar Natesan, Einstein Arulraj, Moorthy Gurunathan. "Sonographic Detection of Intussception with Malrotation: Waughs Syndrome". Journal of Evolution of Medical and Dental Sciences 2015; Vol. 4, Issue 23, March 19; Page: 4040-4045, DOI: 10.14260/jemds/2015/582

ABSTRACT: Intussusception and intestinal malrotation are common causes of intestinal obstruction in infants and children. The two conditions may coexist (Waugh's syndrome) but simultaneous occurrence with midgut volvulus is rare. Malrotation of the gut, a pediatric pathology has been estimated to affect approximately 1 in 500 live births. Malrotation by its nature is associated with mobile right colon, and it might be a prerequisite for intussusception. In this case report, we have discussed that the clinical findings, preoperative imaging are helpful in diagnosing malrotation and midgut volvulus which are associated with intussusception. Thus it aids in choosing the most appropriate surgical approach.

KEYWORDS: Intussusception, malrotation, midgut volvulus.

INTRODUCTION: The association of intussusception with malrotation is referred to as Waugh syndrome.[1] Malrotation occurs in approximately 1 in 500 live births. ${ }^{[2]}$ The incidence of malrotation amongchildren with intussusception is $40 \%$.

We hereby present a case report of Waugh's syndrome associated with midgut volvulus.

CASE REPORT: A 5 month old male child reported to the emergency department with the clinical history of vomiting, abdominal distension, bloody mucoid stools and incessant cry. Routine blood examination revealed $\mathrm{Hb}$ : 12.9 gm\%, TLC: 14500/cu mm, PLT-2. 9lac/cu. mm. Kidney and liver function tests were within normal limits. Ultrasound (US) examination was performed and it revealed dilated fluid-filled small bowel loops with moderate amount of free fluid, Right iliac fossa showed bowel within bowel appearance suggestive of target/pseudo kidney sign of bowel intussusception (Fig. 1. A and B). No pathologic lead point was identified. Transverse ultrasound image through the upper abdomen showed superior mesenteric vein noted to the left of the superior mesenteric artery hence malrotation should be considered (Fig. 2).

In view of surgical emergency non contrast enhanced CT was done and axial image showed target/sausage shaped soft tissue density mass it had alternating areas of low and high attenuation due to bowel wall and mesentry (Fig. 3).

One mergency laparotomy patient was found to have intestinal malrotation with duodenojejunal junction on the right of the midline and 180 mid gut volvulus in clockwise direction. Intussusception with terminal ileum (gangrenous), caecum, appendix, whole of ascending colon, transverse colon were telescoping into descending and sigmoid colon. The volvulus was derotated and the in tussusceptum was reduced. The gangrenous terminal ileum and appendix was resected and Ladd's procedure was done, a diverting ileostomy was created. The patient recovered uneventfully after which an ileo-colonic anastomosis was created (Fig. 4). 


\section{CASE REPORT}

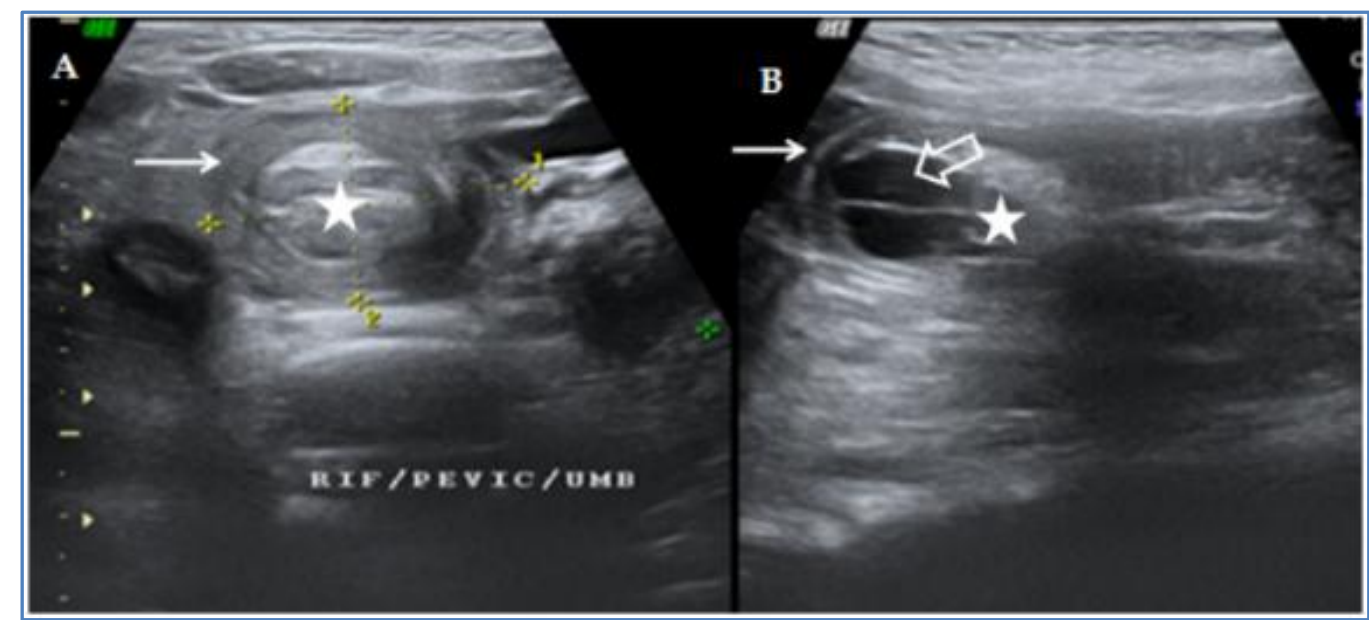

FIG. 1 (A \& B)

Fig. 1(A): Transverse Ultrasound shows a mass with a swirled appearance of alternating hypoechoic and hyperechoic "bowel-within-bowel" appearance (Target sign) in right iliac fossa and umbilical region with central echogenic mesenteryrepresenting intussusception. (B) Longitudinal US of the mass shows sandwich-like appearance of the intussuscipiens (White arrow in $A \& B$ ) and the intussusceptum with mesentry (star-shaped marker in A\&B). Trapped Interloop fluid noted (white open arrow in B).

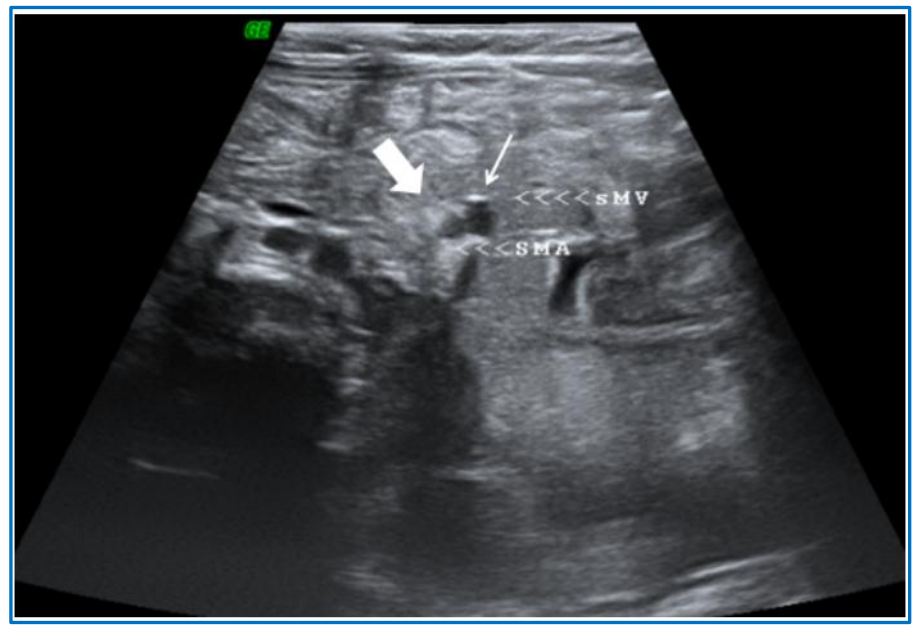

FIG. 2

Fig. 2: Transverse ultrasound image through the upper abdomen shows reversal of the normal superior mesenteric artery SMA (arrowhead) / superior mesenteric vein SMV (White arrow) relationship. 


\section{CASE REPORT}

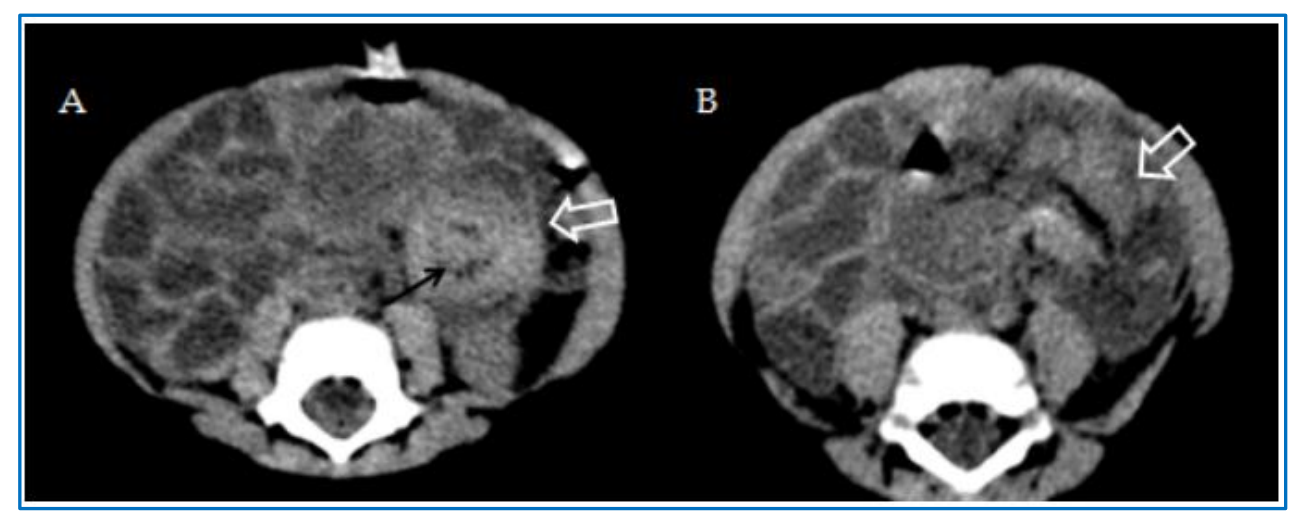

FIG. 3 (A \& B)

Fig. 3 (A \& B): Non contrast enhanced CT scan axial images shows Target shaped (White open arrow in A) /Sausage shaped (White open arrow in B) soft tissue mass with alternating areas of low and high attenuation in left iliac fossa, umbilical, pelvic region of abdomen representing the intussuscepted segment. The fat density (Black arrow in A) seen in the center represent mesenteric fat.

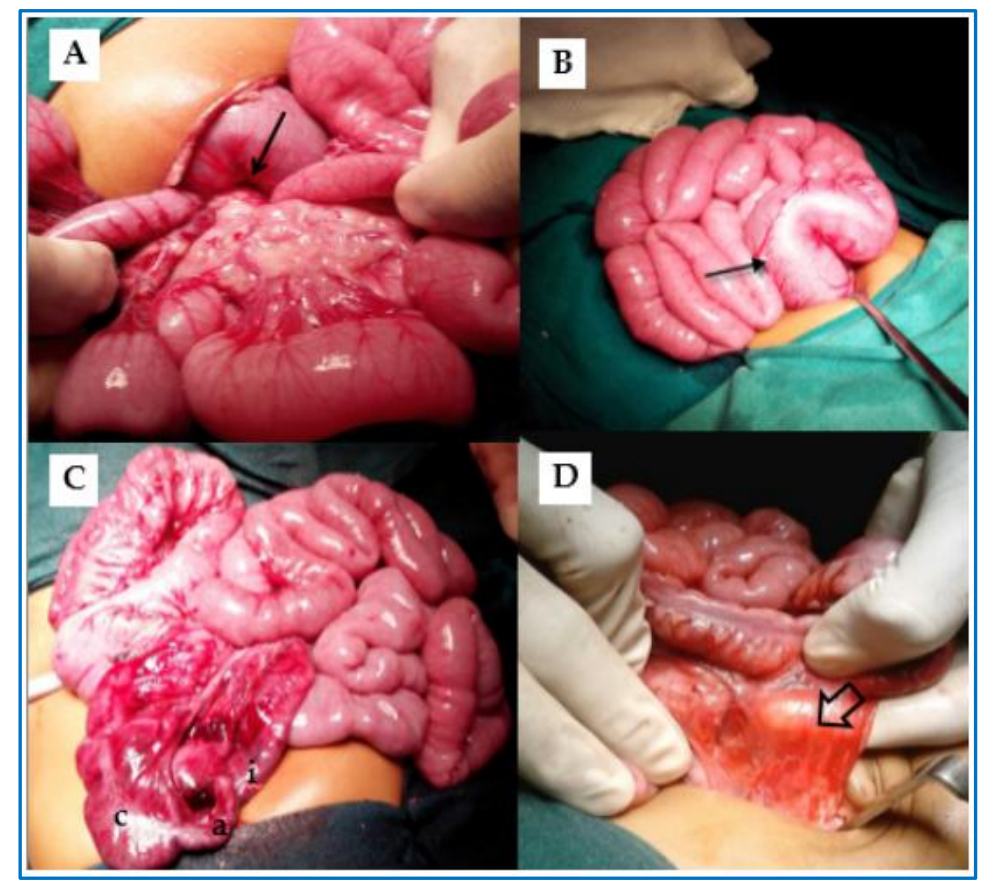

FIG. 4 (A, B, C \& D)

Fig. 4(A): Laparotomy revealed $180^{\circ}$ clockwise midgut volvulus (Black arrow) with ladd's band. (B) An ileocolic intussusception (Black arrow). (C) Appendix (a) and caecum (c) visualized after manual reduction of intussusception with gangreneous distal ileum (i) owing to intussusception. (D) Ladd's band (open black arrow) with narrow mesentry. 


\section{CASE REPORT}

DISCUSSION: The association of intussusception and malrotation has been named as Waugh's Syndrome by Brereton et al,[1] after George E. Waugh, who first described the association in areport in 1911.[3] Intussusception has emerged as one of the common causes of intestinal obstruction in an infant. ${ }^{[4,5]}$ Since then, a very few cases of infantile Waugh's syndrome have been reported in the literature.[6]

It has been suggested that malrotation is often associated with a mobile right colon which may be a pre-requisite for intussusception. ${ }^{[7]}$ The pathophysiology involves easy prolapsing of the ileocolic region into the non-fixed ascending colon found in children with malrotation. Because the ascending colon is not fixed to the retroperitoneum, the intussusceptum advanced into the descending colon and rectum.

The diagnosis of malrotation and volvulus should alwaysbe kept in mind when assessing any infant or childwith symptoms of vomiting and pain, particularly whenthe vomiting is bile-stained.

There are various modalities of imaging technique for detecting intussusception with malrotation and midgut volvulus. They include ultrasonography, Upper gastrointestinal contrast study and CT.

On ultrasonography multiple, concentric, target like appearance of wall layers of invaginated segments (Target sign) on axial scan, as well as pseudokidney sign (sandwich sign) on longitudinal scans were accepted as diagnostic criteria for intussusception. ${ }^{[8]}$ It can assess the relative positions of the SMV and SMA which are mostly abnormal in malrotation. Normally the SMV lies to the right of SMA. This relationship may be abnormal or reversed in malrotation with the SMV anterior or to the left of the SMA. The SMA is identified by pulsality, small size, and a hyperechoic mesenteric collar. ${ }^{[9,10]}$

Other sonographic features such as trapped fluid between the layers of bowel and reduced flow within the mass on color Doppler studies have been associated with a higher failure rate of nonsurgical reduction and increased likelihood of bowel necrosis respectively.[11,12]

Upper gastrointestinal contrast study is the imaging reference standard for diagnosis of malrotation with or without volvulus. Barium sulphate or water soluble contrast medium Gastro graphin can be introduced orally or via nasogastric tube. This is to demonstrate a normal duodenum and duodeno-jejunal (DJ) flexure positionbecause this marks the position of the ligament of Treitz and if this is normally sited then malrotation is effectively excluded. The chief radiographic signs of malrotation on upper GI are:

1. Abnormal position of the duodeno-jejunal junction.

2. Spiral, "corkscrew" or Z-shaped course of the distal duodenum and proximal jejunum, and

3. Location of the proximal jejunum in the right abdomen.[2]

CT is more likely sensitive than sonography in delineating the findings of malrotation. Lack of the duodenum crossing inferior to the SMA from right to left should raise suspicion for malrotation. Abnormalities of situs or organ development may prompt a search for findings of malrotation. As with sonography, CT may show the anomalous orientation of the SMA and superior mesentericvein or the "whirlpool" sign of volvulus.[13,14] In the setting of vascular compromise from malrotation, abnormal perfusion of bowel may also be evident.

For the majority of the reported cases, open laparotomy with manual reduction of the intussusceptum and Ladd's procedure have been the treatment of choice. Waugh's syndrome though a rare entity should be kept in mind during hydrostatic reduction/surgical exploration of intussusception and in cases of recurrent ileocolic intussusception. 


\section{CASE REPORT}

CONCLUSION: The relationship between intestinal malrotation and intussusceptions has been rarely reported in the literature. Waugh's syndrome is missed in cases of closed reduction of intussusception and may be a reason for recurrence. A high degree of clinical suspicion and radiologist's awareness of this entity is helpful in guiding the surgeons towards diagnosis and prevention of morbidity and mortality. If malrotation component is present, non-operative management of intussusception is inadequate because malrotation may cause volvulus.

\section{REFERENCES:}

1. Brereton RJ, Taylor B, Hall CM: Intussusception and malrotation in infants: Waugh's syndrome. Br J Surg 1986; 73: 55-57.

2. Strouse PJ. Disorders of intestinal rotation and fixation ("malrotation"). Pediatr Radiol 2004; 34: 837-851.

3. Waugh GE: Referred penile pain in intussusception with note of three cases. 1911; I: 14921494.

4. Mangete EDO, Allison AB. Intussusception in infancy and childhood: an analysis of 69 cases. West Afr J Med 1994; 13: 87-90.

5. Nmadu PT. The changing pattern of infantile intussusception in Northern Nigeria: a report of 47 cases. Ann Trop Paediatr 1992; 12: 347-350.

6. Baltazar G, Sahyoun C, Sime J, Bitar M, Bitar J, Rao AC. Discovery of a case of Waugh's syndrome during a mission to Haiti. Int J Surg Case Rep. 2012; 3 (1): 22-24.

7. Breckon VM, Hadley GP. Waugh syndrome: Report of six cases. Pediatric Surgery International 2000: 16; 370-373.

8. Ayaz UY, Dilli A, Ayaz S, Api A. Ultrasonographic findings of intussusception in Pediatric cases. Medical ultrasonography. 2011; 13 (4): 272-6.

9. Weinberger E, Winters WD, Liddell RM, et al: Sonographic diagnosis of intestinal malrotation in infants: Importance of the relative positions of the superior mesenteric vein and artery. AJR 1992: 159: 825-828.

10. Dufour D, Dalaet MH, Dassonville M, Cadranel S, Perlmutter N. Midgut malrotation, the reliability of sonographic diagnosis. Pediatr Radiol. 1992; 22: 21-23.

11. Daneman A, Navarro O. Intussusception, Part 1: A review of diagnostic approaches. Pediatric Radiology. 2003; 33: 79-85.

12. Sorantin E, Lindbichler F. Management of intussusception. EurRadiol 2004; 14 (Suppl 4): L14654.

13. Zerin JM, DiPietro MA. Mesenteric vascular anatomy at CT: Normal and abnormal appearances. Radiology 1991; 179: 739-742.

14. Bernstein SM, Russ PD. Midgut volvulus: a rare cause of acute abdomen in an adult patient. AJR 1998; 171: 639-641. 


\section{CASE REPORT}

\section{AUTHORS: \\ 1. Praveen Kumar Natesan \\ 2. Einstein Arulraj \\ 3. Moorthy Gurunathan}

\section{PARTICULARS OF CONTRIBUTORS:}

1. Assistant Professor, Department of Radiology, Chettinad Medical College.

2. Assistant Professor, Department of Radiology, Chettinad Medical College.

3. Associate Professor, Department of Paediatric Surgery, Chettinad Medical College.

FINANCIAL OR OTHER COMPETING INTERESTS: None

\section{NAME ADDRESS EMAIL ID OF THE} CORRESPONDING AUTHOR:

Dr. Praveen Kumar Natesan, Assistant Professor, Department of Radiology, Chettinad University Medical College \& Hospital, Chennai, Tamilnadu, India.

E-mail: drpraveenrd@gmail.com

Date of Submission: 17/02/2015.

Date of Peer Review: 18/02/2015.

Date of Acceptance: 08/03/2015.

Date of Publishing: 19/03/2015. 